\title{
BEAM DYNAMICS SIMULATIONS FOR THE FERMILAB RECYCLER RING BARRIER BUCKETS
}

\author{
H. J. Kang ${ }^{1}$, C. M. Bhat ${ }^{2}$, J. P. Marriner ${ }^{2}$ and J. Maclachlan ${ }^{2}$ \\ ${ }^{1}$ Stanford University, Stanford, CA 94305 USA* \\ ${ }^{2}$ FNAL, Batavia, IL 60510, USA \#
}

\begin{abstract}
The Recycler Ring (RR) is an $8 \mathrm{GeV}$ pbar storage ring for future ppbar collider operations at Fermilab. By design, the beam in the Recycler is stored in three segments (hot, cold and newly transferred beam) azimuthally, using barrier buckets. Properties of the beam in the Recycler are found to be affected by stray magnetic field caused by the Main Injector acceleration ramping process. Here we present results of our measurements of the longitudinal emittance growth of the beam in the Recycler Ring due to the Main Injector ramp and the results of model simulation of this effect using a multi-particle beam dynamics simulation program (ESME).
\end{abstract}

\section{INTRODUCTION}

The purpose of the Recycler [1] at Fermilab is to increase luminosity for the Tevatron experiments by injecting low emittance high intensity antiprotons bunches in each store. The Recycler is also used as a high intensity antiproton accumulator. When the stack size of antiprotons in the Fermilab Accumulator Ring reaches about 40E10 the beam will be transferred to Recycler barrier buckets and stored and cooled in the Recycler. We plan to stack >200E10 antiprotons in the Recycler and cool them over a period of several hours before they are transferred to the Tevatron for ppbar collision. Since antiprotons are stored in the Recycler for an extended time, even a small disturbance of the beam caused by any rf noise, rf base-line shift (or slope) or any external electromagnetic field will result in emittance growth of the beam and may be of concern.

Several measurements indicate that a beam in the Recycler is influenced by stray magnetic fields mainly from magnetic ramping in the MI. The emittance of the beam increases as the beam is exposed to the time varying electromagnetic fields generated during MI ramping. Here an attempt has been made to simulate beam emittance growth. The result of the apparent effect due to the field is qualitatively reproduced by a 2-D beam simulation program (ESME) [2]. The barrier bucket system in the RR has been previously described [3].

\section{THE CHANGE OF BEAM DYNAMICS DUE TO ASTRAY MAGNETIC FIELD}

\section{Initial beam distribution}

The wide band RF system in the RR is capable of generating arbitrary waveforms with a maximum pulse height of $+/-2 \mathrm{kV}$ in each polarity. We use a rectangular barrier pulse of about $908 \mathrm{~ns}$ width. 


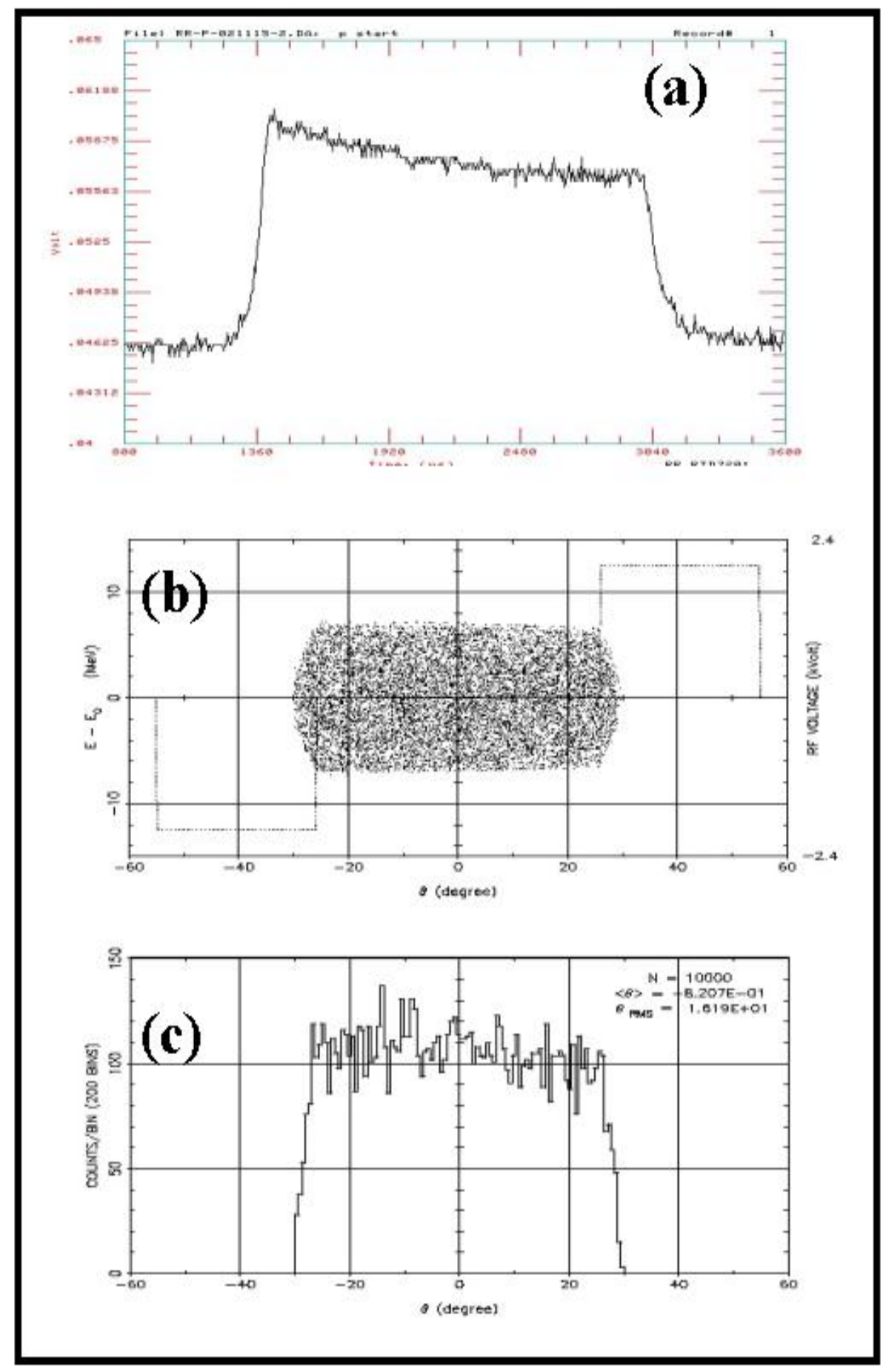

Figure 1: The initial distribution of the beam measured from (a) Wall Current Monitor (WCM) and, (b) and (c) simulation results in $(\Delta \mathrm{E}, \Delta \theta)$-space and its projection on $\theta$-axis 
Ideally the base-line of the middle segment of the barrier bucket should not have any residual voltage, but we observed that there is a shift of the baseline up to $\sim 50 \mathrm{~V}$. The existence of baseline voltage offset causes non-uniformity in the spatial distribution of the beam as shown in Fig.1(a), which is the measured beam distribution from a Wall Current Monitor (WCM). Figs (b) and (c) show simulation results.

\section{Beam distribution with existence of ramping in the MI}

The Recycler and the MI share the same tunnel. The lattice of the both of these synchrotrons are similar at most places except with some small differences at their long straight sections. The MI is designed to accelerate protons from $8 \mathrm{GeV}$ to $120 \mathrm{GeV}$ at a maximum repetition rate of 40 cycles per minutes during pbar stacking and is just about 1.7 meters below the Recycler. At the time of beam transfers from the MI to the Tevatron, the MI ramps from $8 \mathrm{GeV}$ to $150 \mathrm{GeV}$ and the MI dipole magnets saturate for energy above $120 \mathrm{GeV}$. Since the Recycler is very close to the MI, a non-negligible quantity of time varying magnetic field resulting from the MI acceleration penetrates the relatively thin shielding of the Recycler. The growth of the longitudinal emittance is primarily due to nonadiabatic changes in the orbit length of the beam induced by MI ramping. The change in the orbit length causes a shift of the relative position of the beam with respect to the barrier pulses. The shift of the beam position generates inconsistent energy reduction and acceleration for the beam when the beam passes through each barrier pulse, and this causes an increase in the energy spread of the beam and thereby an increase in the longitudinal emittance. We expect that a large longitudinal beam emittance make an even beam distribution throughout $\Delta \theta$. The MI magnetic field also gives a small acceleration and deceleration to the beam through induction.

Fig. 2 presents the beam distribution in $\Delta \theta$ measured at 4 min (Fig.2(a)) and 30 min (Fig.2(b)) in the presence of MI $120 \mathrm{GeV}$ cycles with a repetition rate of about 20cycles/minutes. The Fig. 2 shows wider distributions in $\Delta \theta$ than that shown in Fig. 1(a) (initial distribution). The longitudinal beam emittance corresponding to the beam distribution in Fig.2(b) is found to be $\sim 20 \%$ larger than the one shown in Fig.1(a). The change in energy spread of the beam and the deviation from the initial value $(25 \mathrm{eV}-\mathrm{s})$ of the longitudinal beam emittance as a function of time is shown in Fig. 3(a) and 3(b), respectively. The growth in the longitudinal emittance and the energy spread of the beam is very rapid in the first few minutes after the beam gets injected into the RR, but the growth becomes slower as time increases. 


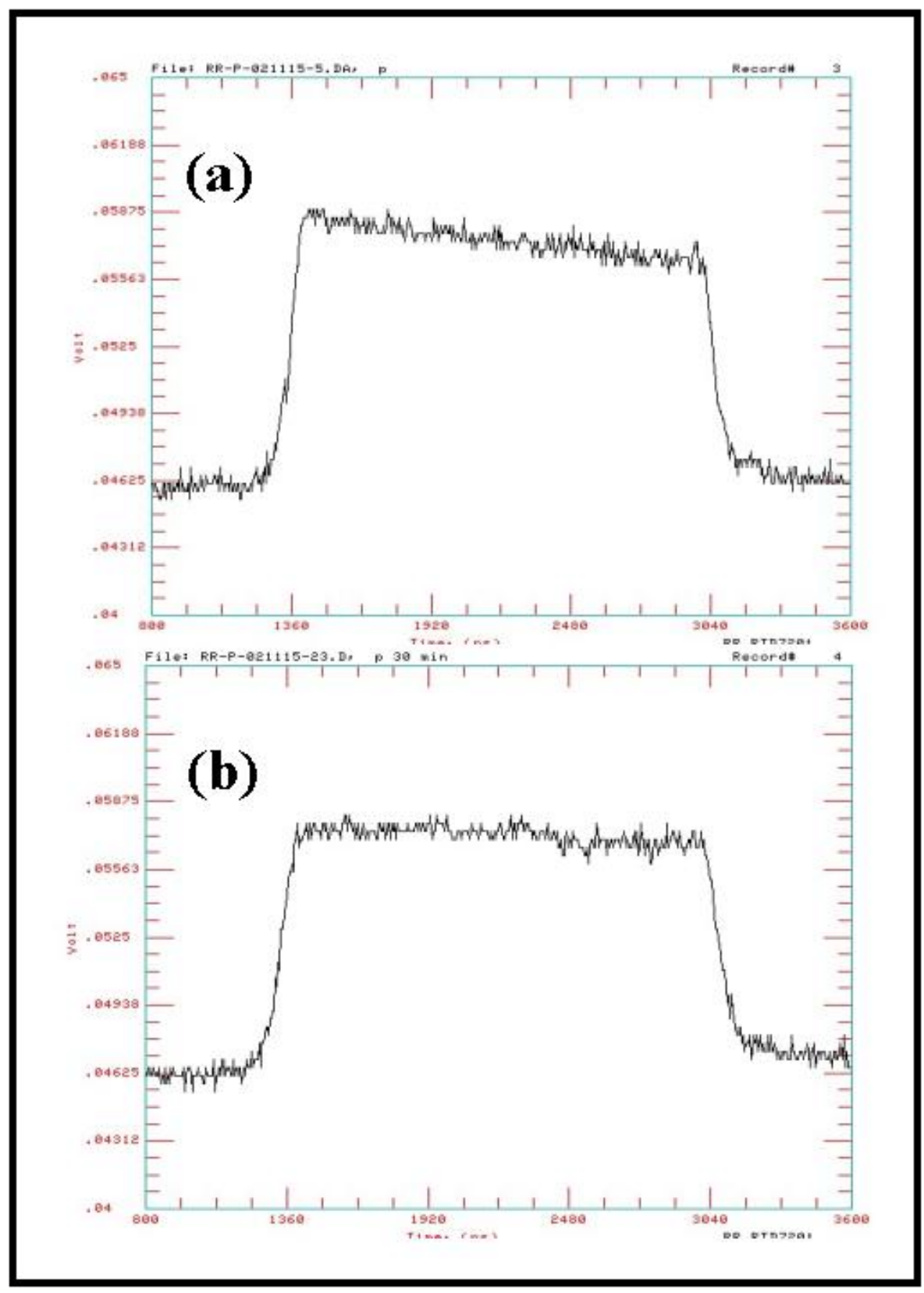

Figure 2: WCM data for the beam after (a) $4 \mathrm{~min}$ and (b) $30 \mathrm{~min}$ later in the presence of $120 \mathrm{GeV}$ MI acceleration cycle at a rate of 20 cycles $/ \mathrm{min}$. 

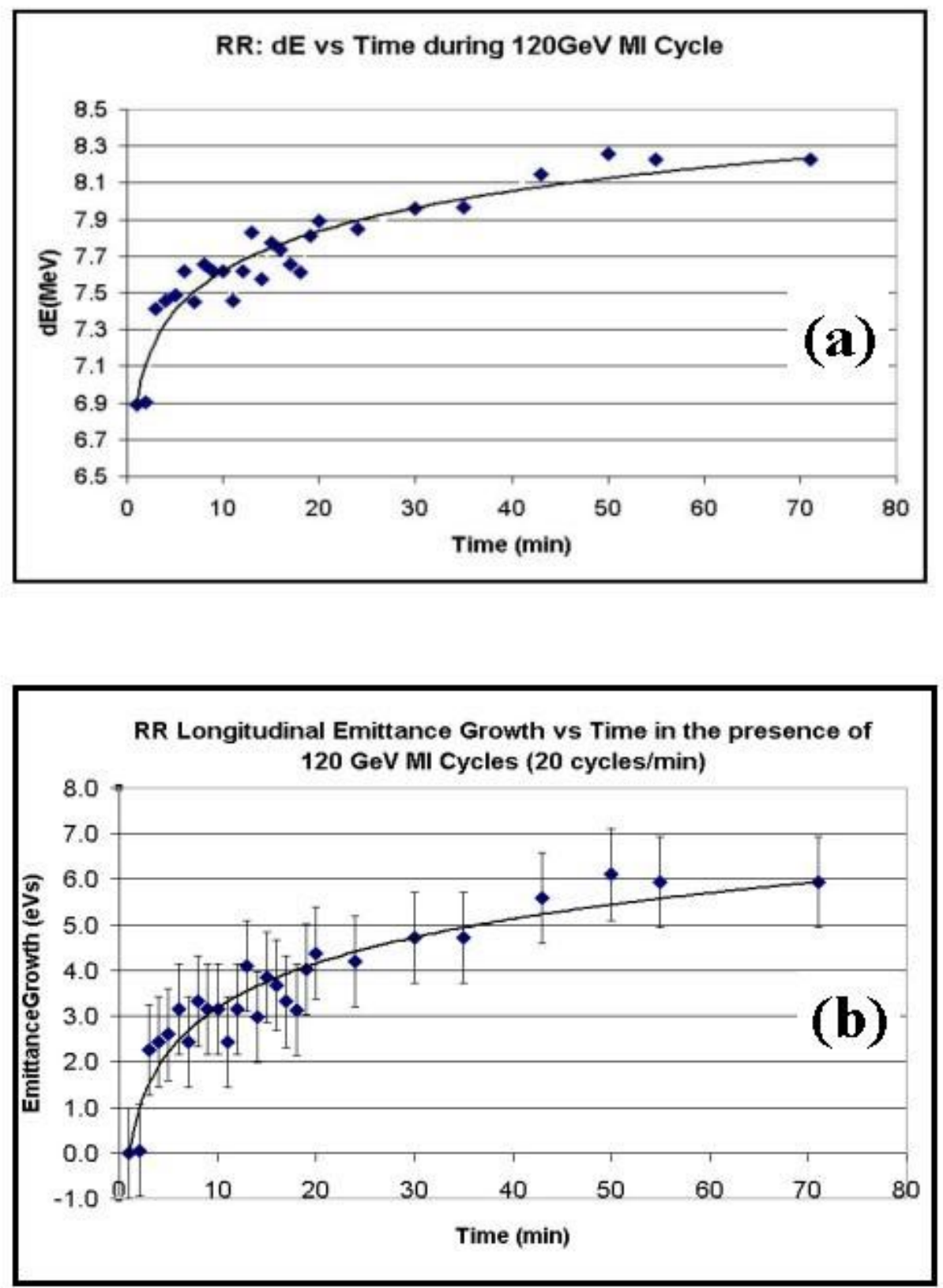

Figure 3: Measured (a) $\Delta \mathrm{E}$ and (b) longitudinal emittance growth of the beam as a function of time in the presence of the MI $120 \mathrm{GeV}$ acceleration cycles

To attempt to simulate the measured beam distribution, we model the effect of MI ramping as if there were an extra time varying voltage in the barrier bucket system instead of a time varying magnetic field. The simulation comprises of increasing and decreasing the voltages of the barrier pulses as well as the baseline by a certain amount during the cycle times of magnetic ramping. The purpose of 
the simulation is to estimate the amount of the extra voltage in the barrier bucket by comparing the WCM data and the predictions from the simulations.
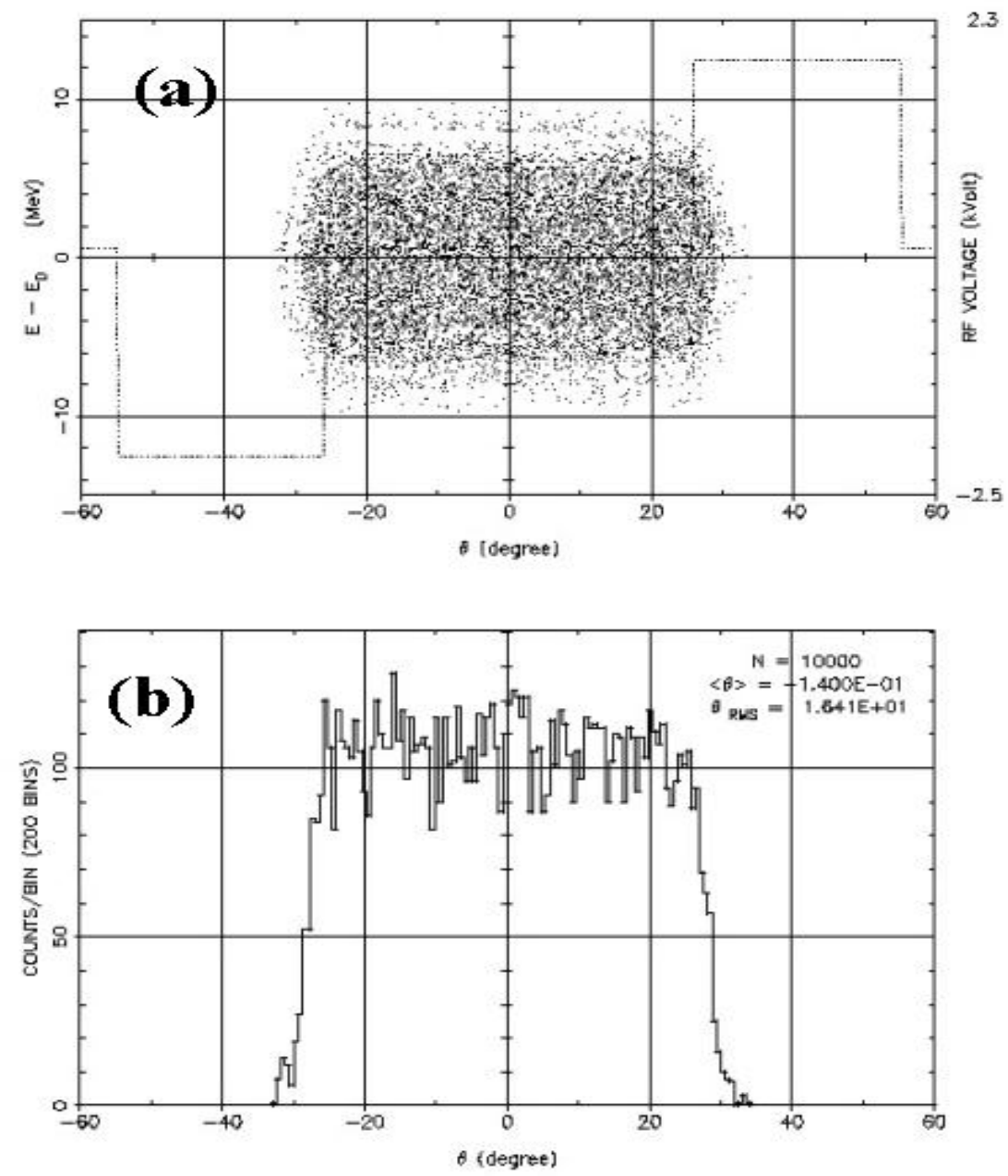

Figure 4: Simulation results for the effects of ramping of the barrier waveform for the first four minutes. Total of 73 ramps in four minutes. The initial distributions for this case are shown in Fig. (b) and (c).

For this paper, the simulation is performed during the first four minutes of MI ramping. The predicted $(\Delta \mathrm{E}, \Delta \theta)$-space distribution of the beam and the projection of the beam in $\Delta \theta$ are presented in Fig.4. The simulation reproduces the shape of the projected beam distribution in $\Delta \theta$ but the rate of emittance growth is not well modelled. The measured growth rate of the distribution in $\Delta \theta$ is $\sim 7 \%$ in 30 minutes and the result from the simulation shows similar amount of increase in the beam distribution. The simulation shows a halo in $(\Delta \mathrm{E}, \Delta \theta)$-space distribution and illustrates that the enlargement of the longitudinal emittance is significantly larger than the the one measured using WCM but the growth of the distribution in $\Delta \theta$ is comparable to the measured value. A plausible explanation for the difference 
between the measured and the simulated value of the growth of longitudinal emittance is that the WCM might not be sensitive enough to detect the extreme wings of the halo of the beam distribution in time $(\Delta \theta)$.

More sophisticated follow-up studies are required with a longer simulation time to attempt to quantitatively reproduce the measurement.

\section{CONCLUSIONS}

Measurements of the longitudinal emittance of proton beams stored in the RR show that there is time dependence of the growth of the emittance in the presence of MI magnetic ramping. The residual field appears to cause a shift of the beam position relative to the barrier pulses. The position shift of the beam is the primary source of the growth of the longitudinal emittance.

We have made an initial attempt to model this emittance growth in a 2-D beam dynamic simulation. The simulated rate of the emittance growth is considerably larger than the observed value.

Further study is required to quantitatively estimate the induced voltage and the rate of emittance growth.

\section{REFERENCES}

* Work supported by the US National Science Foundation (NSF) under grant number PHY0089116.

\# Work supported by the US Department of Energy (DOE) under contract number DE-AC02-76CH03000.

[1] G. Jackson, “The Fermilab Recycler Ring Technical Design Report”, FERMILAB-TM-1991 (1996).

[2] http://www-ap.fnal.gov/ESME; J. MacLachlan and J-F Ostiguy, "Enhancements to the longitudinal dynamics code ESME", IEEE PAC'97 conference proceeding (p.2556)

[3] C.M.Bhat, "Barrier bucket system in the Recyler Ring at Fermilab", $20^{\text {th }}$ ICFA Advanced Beam Dynamics Workshop, 2002. (p.229) 